\title{
Mathematical Reasoning of Treatment Principle Based on "Yin Yang Wu Xing” Theory in Traditional Chinese Medicine (II)
}

\author{
Yingshan Zhang \\ School of Finance and Statistics, East China Normal University, Shanghai, China \\ E-mail:ysh_zhang@163.com \\ Received April 3, 2011; revised April 29, 2011; accepted May 15, 2011
}

\begin{abstract}
By using mathematical reasoning, this paper demonstrates the treatment principle: "Do not treat a disease after it has occurred. But treat the disease before it will occur" (不治已病治未病) based on “Yin Yang Wu Xing" Theory in Traditional Chinese Medicine (TCM). We defined generalized relations and reasoning, introduced the concept of generalized steady multilateral systems, and discussed its energy properties. Later based on the treatment of TCM and treated the healthy body as a steady multilateral system, it has been proved that the treatment principle above is true. The kernel of this paper is the existence and reasoning of the non-compatibility relations in steady multilateral systems, and it accords with the oriental thinking model.
\end{abstract}

Keywords: Traditional Chinese Medicine (TCM), "Yin Yang Wu Xing” Theory, Steady Multilateral Systems, Opposite Relations, Side-Effects, Medical and Drug Resistance Problem

\section{Main Differences between Traditional Chinese Medicine and Western Medicine}

Western medicine treats disease at Microscopic point of view. It is will known that Western medicine always makes directly medical treatments on disease organs after the disease of organs has occurred. The method always destroys the original human being's balance and has none beneficial to human's immunity. Western medicine can produce pollution to human's body, having strong side-effects. Excessively using medicine can easily paralysis the human's immunity, which AIDS is a product of Western medicine. Using medicine too little can easily produce the medical and drug resistance problem.

Traditional Chinese Medicine (TCM) studies the world from the Macroscopic point of view, and its target is in order to maintain the original balance of human being and in order to enhance the immunity. TCM has over 5000-year history. It almost has none side-effects or medical and drug resistance problem. TCM believes that a sick organ will lead other non-disease organ into illness if the sick organ continues to develop. The basic princeple of treatment is to cure the organs before the disease will occur in the organs rather than after. In other words, the basic principle of treatment is "Do not treat a disease after it has occurred. But treat the disease before it will occur” (不治已病治未病).

After long period of practicing, Chinese ancient medical scientists use "Yin Yang Wu Xing" Theory extensively in the traditional treatment to explain the origin of life, human body, pathological changes, clinical diagnosis and prevention. It has become an important part of TCM. "Yin Yang Wu Xing" Theory has a strong influence to the formation and development of Chinese medicine theory. But, many Chinese and foreign scholars still have some questions on the reasoning of TCM.

Zhang's theories, multilateral matrix theory [1] and multilateral system theory [2-15], have given a new and strong mathematical reasoning method from macro (Global) analysis to micro (Local) analysis. He and his colleagues have made some mathematical models and methods of reasoning [1-28], which make the mathematical reasoning of TCM possible based on "Yin Yang Wu Xing" Theory [29]. This paper will use steady multilateral systems to demonstrate the treatment principle of TCM above.

The article proceeds as follows. Section 2 contains basic concepts and main theorems of steady multilateral 
systems while the treatment principle of TCM is demonstrated in Section 3. Some discussions in TCM are given in Section 4 and conclusions are drawn in Section 5.

\section{Basic Concept of Steady Multilateral Systems}

In the real world, we are enlightened from some concepts and phenomena such as "biosphere", "food chain", "ecological balance" etc. With research and practice, by using the theory of multilateral matrices [1], and analyzing the conditions of symmetry [16,17] and orthogonality [18-28] what a stable system must satisfy, in particular, with analyzing the basic conditions what a stable working procedure of good product quality must satisfy $[6,15,22]$, we are inspired and find some rules and methods, then present the logic model of analyzing stability of complex systems-steady multilateral systems [2-15]. There are a number of essential reasoning methods based on the stable logic analysis model, such as "transition reasoning", "atavism reasoning", "genetic reasoning" etc. We start and still use the concepts and notations in papers $[4,5]$.

\subsection{Generalized Relations and Reasoning}

Let $V$ be a non-empty set and define its direct product as $V \times V=\{(x, y): x \in V, y \in V\}$. A non-empty subset $R \subset$ $V \times V$ is called a relation of $V$. Traditional Chinese Science (TCS) mainly researches general relation rules for general $V$ rather than for special $V$.

For a relation class $\mathfrak{R}=\left\{R_{0}, \cdots, R_{m-1}\right\}$, define both an inverse relation $R_{i}^{-1}$ of $R_{i} \in \mathfrak{R}$ and a relation multiplication $R_{i} * R_{j}$ between $R_{i} \in \mathfrak{R}$ and $R_{j} \in \mathfrak{R}$ as follows:

$$
R_{i}^{-1}=\left\{(x, y):(y, x) \in R_{i}\right\}
$$

$R_{i} * R_{j}=\{(x, y):$ there exists at least an $u \in V$ such that $(x, u) \in R_{i}$ and $\left.(u, y) \in R_{j}\right\}$.

The relation $R_{i}$ is called reasonable if $R_{i}^{-1} \in \mathfrak{R}$. A generalized reasoning of $V$ is defined as for $R_{i} * R_{j} \neq \varnothing$ there is a relation $R_{k} \in \mathfrak{R}$ such that $R_{i} * R_{j} \subset R_{k}$.

\subsection{Equivalence Relations}

Let $V$ be a non-empty set with a relation $R$. The relation $R$ is called an equivalence relation, denoted by $\sim$, if the following three conditions are all true:

(1) Reflexive: $(x, x) \in R$ for all $x \in V$, i.e., $x \sim x$;

(2) Symmetric: if $(x, y) \in R$, then $(y, x) \in R$, i.e., if $x \sim y$, then $y \sim x$;

(3) Conveyable (Transitivity): if $(x, y) \in R,(y, z) \in R$, then $(x, z) \in R$, i.e., if $x \sim y, y \sim z$, then $x \sim z$.

Furthermore, the relation $R$ is called a compatibility relation if there exists a non-empty subset $R_{1} \subset R$ such that $R_{1}$ satisfies at least one of the conditions above. And the relation $R$ is called a non-compatibility relation if there doesn't exist any non-empty subset $R_{1} \subset R$ such that $R_{1}$ satisfies any one of the conditions above. Any one of compatibility relations can be expanded into an equivalence relation [2].

Western science only considers the reasoning under one Axiom system such that only compatibility relation reasoning is researched. However there are many Axiom systems in Nature. TCS mainly researches the generalized reasoning among many Axiom systems in Nature. Of course, she also considers the reasoning under one Axiom system but she only expands the reasoning as the equivalence relation reasoning.

\subsection{Two Kinds of Opposite Non-Compatibility Relations}

Equivalence relations, even compatibility relations, can not portray the structure of the complex systems clearly. In the following, we consider two non-compatibility relations.

For example, let $V=V_{0}+\cdots+V_{4}$ be a non-empty, there are two kinds of opposite relations: the neighboring relation $R_{1}$, denoted $\rightarrow$ and the alternate relation $R_{2}$, denoted $\Rightarrow$, having the property:

(1) If $x \rightarrow y, y \rightarrow z$, then $x \Rightarrow z$, i.e., if $(x, y) \in R_{1}$, $(y, z) \in R_{1}$, then $(x, z) \in R_{2}$, or, $R_{1} * R_{1}=R_{2}$;

$\Leftrightarrow$ if $x \rightarrow y, x \Rightarrow z$, then $y \rightarrow z$, i.e., if $(x, y) \in R_{1}$, $(x, z) \in R_{2}$, then $(y, z) \in R_{1}$, or, $R_{1}^{-1} * R_{2}=R_{1}$;

$\Leftrightarrow$ if $x \Rightarrow z, y \rightarrow z$, then $x \rightarrow y$, i.e., if $(x, z) \in R_{2}$, $(y, z) \in R_{1}$, then $(x, y) \in R_{1}$ or, $R_{2} * R_{1}^{-1}=R_{1}$.

(2) If $x \Rightarrow y, y \Rightarrow z$, then $z \rightarrow x$, i.e., if $(x, y) \in R_{2}$, $(y, z) \in R_{2}$, then $(z, x) \in R_{1}$,or, $R_{2} * R_{2}=R_{1}^{-1}$;

$\Leftrightarrow$ if $z \rightarrow x, x \Rightarrow y$, then $y \Rightarrow z$,i.e., if $(z, x) \in R_{1}$, $(x, y) \in R_{2}$, then $(y, z) \in R_{2}$, or, $R_{1} * R_{2}=R_{2}^{-1}$;

$\Leftrightarrow$ if $y \Rightarrow z, z \rightarrow x$, then $x \Rightarrow y$,i.e., if $(y, z) \in R_{2}$, $(z, x) \in R_{1}$, then $(x, y) \in R_{2}$, or, $R_{2} * R_{1}=R_{2}^{-1}$.

Two kinds of opposite relations can not be existence separately. Such reasoning can be expressed in Figure 1. The first triangle reasoning is known as a jumping-transition reasoning, while the second triangle reasoning is known as an atavism reasoning. Reasoning method is a triangle on both sides decided to any third side. Both neighboring relations and alternate relations are non-compatibility relations (of course, non-equivalence relations), which are called two kinds of opposite non-compatibility relations.

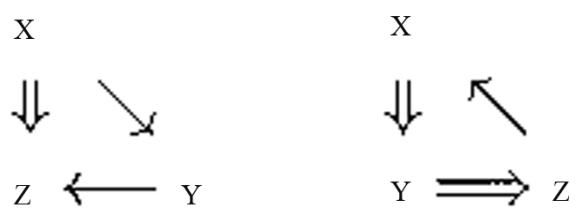

Figure 1. Triangle reasoning. 


\subsection{Genetic Reasoning}

Let $V$ be a non-empty set with an equivalence relation $R_{0}$, a neighboring relation $R_{1}$ and some alternate relations $R_{2}$. Then a genetic reasoning is defined as follows:

(1) if $x \sim y, y \rightarrow z$, then $x \rightarrow z$, i.e., if $(x, y) \in R_{0}$, $(y, z) \in R_{1}$, then $(x, z) \in R_{1}$, or, $R_{0} * R_{1}=R_{1}$;

(2) if $x \rightarrow y, y \sim z$, then $x \rightarrow z$, i.e., if $(x, y) \in R_{1}$, $(y, z) \in R_{0}$, then $(x, z) \in R_{1}$, or, $R_{1} * R_{0}=R_{1}$;

(3) if $x \sim y, y \Rightarrow z$, then $x \Rightarrow z$, i.e., if $(x, y) \in R_{0}$, $(y, z) \in R_{2}$, then $(x, z) \in R_{2}$, or, $R_{0} * R_{2}=R_{2}$;

(4) if $x \Rightarrow y, y \sim z$, then, $x \Rightarrow z$ i.e., if $(x, y) \in R_{2}$, $(y, z) \in R_{0}$, then $(x, z) \in R_{2}$, or, $R_{2} * R_{0}=R_{2}$.

\subsection{Multilateral Systems}

For a non-empty set $V$ and its some relations,

$$
R_{0}, \cdots, R_{m-1} \subset V \times V=\{(x, y): x \in V, y \in V\},
$$

Denote $\mathfrak{R}=\left\{R_{0}, \cdots, R_{m-1}\right\}$. Then the form $(V, \mathfrak{R})$ (or simply, $V)$ is called a multilateral system $[3,13]$, if $(V, \mathfrak{R})$ satisfies the following properties:

a) $R_{0}+\cdots+R_{m-1} \subset V \times V$.

b) $R_{0} * R_{i}=R_{i} * R_{0}=R_{i}, \forall i$.

c) For any $R_{i} \in \mathfrak{R}=\left\{R_{0}, \cdots, R_{m-1}\right\}$, we have $R_{i}^{-1} \in \mathfrak{R}$.

d) For any $R_{k} * R_{l} \neq \varnothing$, there exists $R_{i}$ such that $R_{k} * R_{l} \subset R_{i}$.

The $d$ ) is called the generalized reasoning, the $a$ ) the uniqueness of reasoning, the $b$ ) the hereditary of reasoning (or genetic reasoning) and the $c$ ) the condition of reasoning, respectively. In this case, the two-relation set $\left\{R_{i}, R_{i}^{-1}\right\}$ is a lateral relation of $V$. Furthermore, the $V$ and $\mathfrak{R}$ are called a state space and relation classes of $(V, \mathfrak{R})$, respectively. The generalized $\operatorname{system}(V, \mathfrak{R})$ can be written as $\left(V_{0}+\cdots+V_{n-1},\left\{R_{0}, \cdots, R_{m-1}\right\}\right)$.

For a multilateral $\operatorname{system}(V, \mathfrak{R})$, it is called complete (or, perfect) if " $\subset$ " changes into "=".And it is called complex if there exists at least a non-compatibility relation $R_{i} \in \mathfrak{R}$. In this case, the multilateral $\operatorname{system}(V, \mathfrak{R})$ is also called a logic analysis model of complex systems [2-15].

For a multilateral system $(V, \mathfrak{R})$, assume that there exist relations: an equivalence relation, a neighboring relation, and an alternate relation in system $V$, which satisfy genetic reasoning. Suppose that for every $x, y \in V$, at least there is one of the three relations between $x$ and $y$, and there are not $x \rightarrow y$ and $x \Rightarrow y$ at the same time, i.e., $\quad V \times V \subset R_{0} \cup R_{1} \cup R_{2} \cup R_{2}^{-1} \cup R_{1}^{-1}, \quad R_{1} \cap R_{2}=\varnothing$. Then it is easily to prove that $V$ is a multilateral system with two non-compatibility relations, i.e., $(V, R)=$ $\left(V,\left\{R_{0}, R_{1}, \cdots, R_{4}\right\}\right)$, where two non-compatibility relations are the lateral relations $\left\{R_{1}, R_{1}^{-1}=R_{4}\right\}$ and $\left\{R_{2}, R_{2}^{-1}=R_{3}\right\}$ satisfying $R_{i} * R_{j}=R_{\bmod (i+j, 5)}, \forall i, j$.
The multilateral $\operatorname{system}(V, \mathfrak{R})$ is equivalent to the logic architecture of reasoning model of "Yin Yang" Theory in Ancient China. In this paper, we only consider this multilateral system.

Theorem 2.1. For a multilateral system $(V, \mathfrak{R})$ with two non-compatibility relations, $\forall x, y \in V$, only one of the following five relations is existent and correct: $x \sim y$, $x \rightarrow y, x \leftarrow y, x \Rightarrow y, x \Leftarrow y$ i.e., $\quad V \times V=R_{0}+R_{1}+R_{2}$ $+R_{3}+R_{4}$.

Theorem 2.2. For a multilateral system $(V, \mathfrak{R})$ with two non-compatibility relations, $\forall x, y, z \in V$, the following reasoning holds.

(1) If $x \rightarrow z, y \rightarrow z$, then $x \sim y$, i.e., $R_{1} * R_{1}^{-1}=R_{0}$;

(2) if $x \Rightarrow z, y \Rightarrow z$, then $x \sim y$,i.e., $R_{2} * R_{2}^{-1}=R_{0}$;

(3) if $x \rightarrow y, x \rightarrow z$, then $y \sim z$,i.e., $R_{1}^{-1} * R_{1}=R_{0}$;

(4) if $x \Rightarrow y, x \Rightarrow z$, then $y \sim z$,i.e., $R_{2}^{-1} * R_{2}=R_{0}$.

\subsection{Steady Multilateral Systems}

The multilateral system $V$ with two non-compatibility relations is said steady (or, stable) if there exists at least the chain $x_{1}, \cdots, x_{n} \in V$, which satisfies any one of the two conditions below:

$x_{1} \rightarrow x_{2} \rightarrow \cdots \rightarrow x_{n} \rightarrow x_{1}$, i.e., there exists a number $n$ such that $R_{1}^{* n} \cap R_{0} \neq \varnothing$ where $R_{1}^{* n}=R_{1} * \cdots * R_{1}$;

$x_{1} \Rightarrow x_{2} \Rightarrow \cdots \Rightarrow x_{n} \Rightarrow x_{1}$, i.e., there exists a number $n$ such that $R_{2}^{* n} \cap R_{0} \neq \varnothing$ where $R_{2}^{* n}=R_{2} * \cdots * R_{2}$.

Theorem 2.3. For a steady multilateral system $(V, \mathfrak{R})$ $=\left(V_{0}+\cdots+V_{n-1},\left\{R_{0}, \cdots R_{m-1}\right\}\right)$ with two non-compatibility relations, there exists five-length chain, and the length of the chain is integer times of 5, i.e., there exists $R_{1}^{* 5} \cap R_{0} \neq \varnothing \quad\left(\right.$ or $\left.\quad R_{2}^{* 5} \cap R_{0} \neq \varnothing \quad\right) \quad$ and $n=5 k \quad$ if $R_{1}^{* n} \cap R_{0} \neq \varnothing \quad\left(\right.$ or $\left.R_{2}^{* n} \bigcap R_{0} \neq \varnothing\right)$.

Theorem 2.4. For a steady multilateral system $(V, \mathfrak{R})$ $=\left(V_{0}+\cdots+V_{n-1},\left\{R_{0}, \cdots R_{m-1}\right\}\right)$ with two non-compatibility relations, assume there exists a chain $\left\{x_{0}, x_{1}, x_{2}\right.$, $\left.x_{3}, x_{4}\right\}$, then $n=m=5$. In other words, $m=5$ and there exists a partition of $V$ as follows:

$$
\begin{gathered}
V=V_{0}+V_{1}+V_{2}+V_{3}+V_{4}, \\
V_{i}=\left\{y \in V \mid y \sim x_{i}\right\}, \forall i=0, \cdots, 4 .
\end{gathered}
$$

Theorem 2.5. For the decomposition above for the steady multilateral system with two non-compatibility relations, there exist relations below Figure 2. In other words, $R_{1}^{* 5}=R_{0}=R_{2}^{* 5}$, i.e., $(V, \mathfrak{R})$ is a complete multilateral system.

Theorem 2.6. For each element $x \in V$ in a steady multilateral system $V$ with two non-compatibility relations, there exist five equivalence classes below: 


$$
\begin{gathered}
X=\{y \in V \mid y \sim x\}, \quad X_{S}=\{y \in V \mid x \rightarrow y\}, \\
X_{K}=\{y \in V \mid x \Rightarrow y\}, K_{X}=\{y \in V \mid y \Rightarrow x\}, \\
S_{X}=\{y \in V \mid y \rightarrow x\}
\end{gathered}
$$

which the five equivalence classes have relations below Figure 3.

These theorems can been found in [2-15]. Figures 2 and 3 in Theorems 2.5 and 2.6 are the Figures of " $W u$ Xing" Theory in Ancient China. The steady multilateral system $V$ with two non-compatibility relations is equivalent to the logic architecture of reasoning model of "Yin Yang Wu Xing" Theory in Ancient China. What describes the general method of complex systems can be used in human complex systems

\section{Relationship Analysis of Steady Multilateral Systems}

\subsection{Energy Theory of Multilateral Systems}

Western Medicine is different from TCM because the TCM has a concept of Chi (or $Q i$, 气) as a form of energy. It is believed that this energy exists in all things (living and non-living) including air, water, food and sunlight. Chi is said to be the unseen vital force that

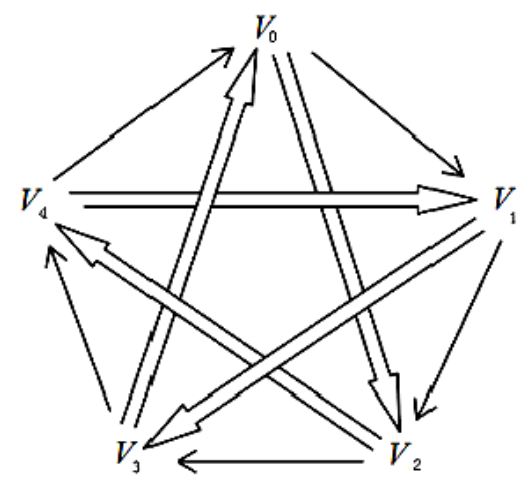

Figure 2. Uniquely steady architecture of Wu-Xing.

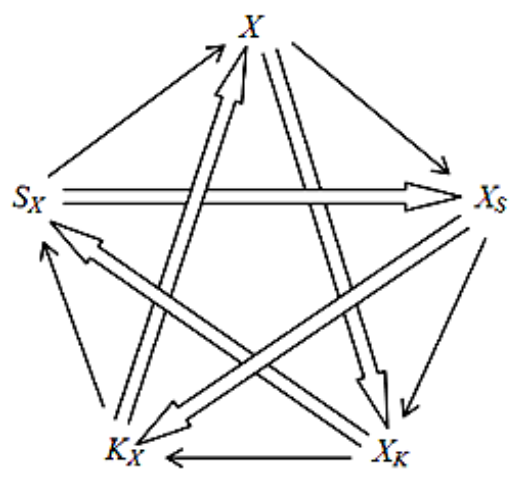

Figure 3. The method of finding Wu-Xing. nourishes one's body and sustains one's life. It is also believed that an individual is born with an original amount of Chi at the beginning of one's life and as one grows and lives, one acquires $C h i$ from eating and drinking, from breathing the surrounding air and also from living in one's environment. And the one also affords Chi for the human body's meridian system (Jing-Luo (经络)) and Zang Xiang (藏象) organs which form a parasitic system of human, called the second physiological system of human. The second system of human controls the first physiological system (Anatomy system) of human. An individual would become ill or dies if one's $C h i$ in the body is imbalanced or exhausted. The concept is summarized as the energy theory of multilateral systems.

In mathematics, a multilateral system is said to have Energy (or Dynamic) if there is a non-negative function $\varphi(*)$ which makes every subsystem meaningful of the multilateral system. Similarly to papers $[4,5]$, unless stated otherwise, any equivalence relation is the liking relation, any neighboring relation is the loving relation, and any alternate relation is the killing relation.

Suppose $V$ is a steady multilateral system having energy during normal operation. Then its energy function for any subsystem of the multilateral system has a center (or average, expected value, median in Statistics). And this state is called normal when the energy function is nearly to the center. Normal state is the better state.

A subsystem of a multilateral system is called not running properly (or disease, abnormal) if the energy deviation from the center of the subsystems is too large, the high (real disease) or the low (virtual disease).

In general, a disease is less serious if it satisfies both the loving relation and the killing relation of the multilateral system. This disease is less serious because this disease has not undermine the balance of the multilateral system and the normal order, which makes the intervention did not reduce the intervention reaction coefficient $\rho_{1}$ (See [4,5]). But the disease is serious if it doesn't satisfy both the loving relation and the killing relation of the multilateral system, i.e., there is an incest order. This disease is serious because the disease has destroyed the balance and the normal order of the multilateral system, which makes the intervention reaction coefficient $\rho_{1}$ reduced response to intervention. For a steady multilateral system $V$ with two non-compatibility relations, suppose that the subsystems $X, X_{S}, X_{K}, K_{X}, S_{X}$ are the same as those defined in Theorem 2.6. Then the diseases can be decomposed into the following classes:

Definition 3.1. (involving(相及) and infringing upon (相犯)) Suppose a disease occurred between $X$ and $X_{S}$.

The disease is called less serious if $X$ is virtual disease and so is $X_{S}$ at the same time (Mother disease involv- 
ing the son 母病及子), or, if $X$ is real disease and so is $X_{S}$ at the same time (Son disease infringing upon the mother 子病犯母).

The disease is called rare disease if $X$ is real disease but $X_{S}$ is virtual disease at the same time, or if $X$ is virtual disease and $X_{S}$ is real disease at the same time.

Definition 3.2. (multiplying(相乘) and insulting(相侮)) Suppose a disease occurred between $X$ and $X_{K}$.

The disease is called less serious if $X$ is real disease and $X_{K}$ is virtual disease at the same time. The relation between $X$ and $X_{K}$ is called multiplying-relation (相乘).

The disease is called serious if $X$ is virtual disease but $X_{K}$ is real disease at the same time. The relation between $X$ and $X_{K}$ is called insulting-relation (相侮). It means that $X_{K}$ has harmed the $X$ by using the method of incest.

The disease is called rare disease if $X$ is real disease and so is $X_{K}$ at the same time, or if $X$ is virtual disease and so is $X_{K}$ at the same time.

The disease is called more serious if $X$ is real disease, and $X_{K}$ is virtual disease but $K_{X}$ is also virtual disease at same time, i.e., $X$ not only multiplies in $X_{K}$, but also $X$ insults $K_{X}$ by using the method of incest. It is because the energy of $X$ is too high. The relation between $X$ and $X_{K}$ is also called multiplying-insulting relation (相乘致侮).

The disease of multiplying-insulting relation will result in more than three subsystems falling-ill. Generally, three or more subsystems falling-ill, it will be hard to cure. Therefore, the multiplying-insulting disease should be avoided as much as possible. In Chinese words, it is that "Again and again, not only to the repeated to four “(只有再一再二, 没有再三再四)一Allow one or two subsystems fall ill, but don't allow three or four subsystems fall ill.

Theorem 3.1. The occurrence of disease has its laws: The first occurrence of the loving relation and the killing relation after the disease. In other words, the following statements are true.

If a subsystem $X$ of a multilateral system $V$ falls virtual disease, the law is the first occurrence of the less serious virtual disease of the mother $S_{X}$, and the less serious real disease of the bane $K_{X}$ after the disease, and next the more serious real disease of the prisoner $X_{K}$, and finally the less serious virtual disease of the son $X_{S}$.

If a subsystem $X$ of a multilateral system $V$ falls real disease, the law is the first occurrence of the less serious real disease of the son $X_{S}$, and the less serious virtual disease of the prisoner $X_{K}$ after the disease, and next the more serious virtual disease of the bane $K_{X}$, and finally the less serious real disease of the mother $S_{X}$.

It is because, by Theorem 3.3 below, the energy of the son $X_{S}$ or the mother $S_{X}$ of a falling-ill subsystem $X$ of a multilateral system $V$ is firstly changed by following the energy change of $X$ corresponding to the real or virtual disease, respectively. If the disease continues to develop, it will lead to undermine the capability of self-protection of the multilateral system, i.e., the selfprotection coefficient $\rho_{3}$ will near to small. By Theorem 3.2 below, the victim $X_{K}$ or $K_{X}$ of $X$ will encounter the sick which is different from the disease direction of $X$.

In a subsystem of a multilateral system being not running properly, if this sub-system energy increases or decreases through external force, making the energy return to the center (or average, expected value, median), this method is called intervention(or making a medical treatment) to the multilateral system.

The purpose of intervention is to make the multilateral system return to normal state. The method of intervenetion is to increase or decrease the energy of a subsystem.

What kind of treatment should follow the principle to treat it? For example, Western medicine always makes directly medical treatments on disease organs after the disease of organs has occurred. But TCM always makes indirectly medical treatments on disease organs before the disease of organs will occur. In mathematics, which is more reasonable?

Based on this idea, many issues are worth further discussion. For example, if an intervention treatment has been done to a non-sick subsystem of a multilateral system, what situation will happen?

\subsection{Intervention Rule of a Multilateral System}

For a steady multilateral system $V$ with two non-compatibility relations, suppose that there is an external force (or an intervening force) on the subsystem $X$ of $V$ which makes the energy $\varphi(X)$ changed by increment $\Delta \varphi(X)$, then the energies $\varphi\left(X_{S}\right), \varphi\left(X_{K}\right), \varphi\left(K_{X}\right), \varphi\left(S_{X}\right)$ of other subsystems $X_{S}, X_{K}, K_{X}, S_{X}$ (defined in Theorem 2.6) of $V$ will be changed by the increments $\Delta \varphi\left(X_{S}\right)$, $\Delta \varphi\left(X_{K}\right), \Delta \varphi\left(K_{X}\right), \Delta \varphi\left(S_{X}\right)$, respectively.

The concepts of the capability of intervention reaction, beneficiaries and victims come from papers $[4,5]$. In general, the intervention rule is similar to force and reaction in Physics. In other words, if a subsystem of multilateral system $V$ has been intervened, then the energy of subsystem which has neighboring relation ( or beneficiary ) changes in the same direction of the force, and the energy of subsystem which has alternate relation (or victim ) changes in the opposite direction of the force. The size of the energy changed is equal, but the direction opposite.

In mathematics, the changing laws of intervention rule 
are as follows.

(1) If $\Delta \varphi(X)=\Delta>0$, then $\Delta \varphi\left(X_{S}\right)=\rho_{1} \Delta, \Delta \varphi$ $\left(X_{K}\right)=-\rho_{1} \Delta, \Delta \varphi\left(K_{X}\right)=-\rho_{2} \Delta, \Delta \varphi\left(S_{X}\right)=\rho_{2} \Delta$;

(2) If $\Delta \varphi(X)=-\Delta<0$, then $\Delta \varphi\left(X_{S}\right)=-\rho_{2} \Delta, \Delta \varphi$ $\left(X_{K}\right)=\rho_{2} \Delta, \Delta \varphi\left(K_{X}\right)=\rho_{1} \Delta, \Delta \varphi\left(S_{X}\right)=-\rho_{1} \Delta ;$ where $1 \geq \rho_{1} \geq \rho_{2} \geq 0$. Both $\rho_{1}$ and $\rho_{2}$ are called intervention reaction coefficients, which are used to represent the capability of intervention reaction. The larger $\rho_{1}$, the better the capability of intervention reaction. The state $\rho_{1}=1$ is the best state but the state $\rho_{1}=0$ is the worst state.

Medical and drug resistance problem is that such a question, beginning more appropriate medical treatment, but is no longer valid after a period. It is because the capability of intervention reaction is bad, i.e., the intervention reaction coefficients $\rho_{1}$ is too small. In the state $\rho_{1}=1$, any medical and drug resistance problem is nonexistence but in the state $\rho_{1}=0$, medical and drug resistance problem is always existence. At this point, the paper advocates the principle of treatment to avoid medical and drug resistance problems.

\subsection{Self-Protection Rule of a Multilateral System}

If there is an intervening force on the subsystem $X$ of a steady multilateral system $V$ which makes the energy $\varphi(X)$ be changed by increment $\Delta \varphi(X)$ such that the energies $\varphi\left(X_{S}\right), \varphi\left(X_{K}\right), \varphi\left(K_{X}\right), \varphi\left(S_{X}\right)$ of other subsystems $X_{S}, X_{K}, K_{X}, S_{X}$ (defined in Theorem 2.6) of $V$ will be changed by the increments $\Delta \varphi\left(X_{S}\right)$, $\Delta \varphi\left(X_{K}\right), \Delta \varphi\left(K_{X}\right), \Delta \varphi\left(S_{X}\right)$, respectively, then can the multilateral system $V$ have capability to protect the worst victim to restore?

The concepts of the general capability of self-protecttion, the better capability of self-protection and worst victims come from papers [4,5]. In general, there is an essential principle of self-protection: any harmful subsystem of $X$ should be protected by using the same intervention force but any beneficial subsystem of $X$ should not if the intervention treatment has been done on the subsystem $X$ of the multilateral system.

In order to represent the capability of self-protection, the concept of the self-protection coefficient $\rho_{3}$ is also need. The larger $\rho_{3}$, the better the capability of self-protection. The state $\rho_{3}=\rho_{1}$ is the best state but the state $\rho_{3}=\rho_{1}=0$ is the worst state, where $\rho_{1}$ is the intervention reaction coefficient.

In the case of virtual disease, the treatment method of intervention is to increase the energy. If the treatment has been done on $X$ by the increment $\Delta \varphi(X)=\Delta>0$, the worst victim is the prisoner $X_{K}$ of $X$ which has the increment $-\rho_{1} \Delta$. Thus, the treatment of self-protection is to restore the prisoner $X_{K}$ of $X$ and the restoring method of self-protection is to increase the energy $\varphi\left(X_{K}\right)$ of the prisoner $X_{K}$ of $X$ by using the intervention force on $X$ according to the intervention rule. But there may be an increase degree which is $\rho_{3} \Delta$ rather than $\rho_{1} \Delta$ if the capability of self-protection $\rho_{3}$ is less than $\rho_{1}$.

In the case of real disease, the treatment method of intervention is to decrease the energy. If the treatment has been done on $X$ by the increment $\Delta \varphi(X)=-\Delta<0$, the worst victim is the bane $K_{X}$ of $X$ which has the increment $\rho_{1} \Delta$. Thus, the treatment of self-protection is to restore the bane $K_{X}$ of $X$ and the restoring method of self-protection is to decrease the energy $\varphi\left(K_{X}\right)$ of the bane $K_{X}$ of $X$ by using the same intervention force on $X$ according to the intervention rule. But there may be an decrease degree which is $-\rho_{3} \Delta$ rather than $-\rho_{1} \Delta$ if the capability of self-protection $\rho_{3}$ is less than $\rho_{1}$.

In mathematics, the following self-protection laws hold.

(1) If $\Delta \varphi(X)=\Delta>0$, then the energy of subsystem $X_{K}$ will decrease the increment $\left(-\rho_{1} \Delta\right)<0$, which is the worst victim. So the capability of self-protection increases the energy of subsystem $X_{K}$ by increment $\left(\rho_{3} \Delta\right)$ in order to restore the worst victim $X_{K}$ by according to the intervention rule.

(2) If $\Delta \varphi(X)=-\Delta<0$, then the energy of subsystem $K_{X}$ will increase the increment $\left(\rho_{1} \Delta\right)$, which is the worst victim. So the capability of self-protection decreases the energy of subsystem $K_{X}$ by increment $\left(-\rho_{3} \Delta\right)$ in order to restore the worst victim $K_{X}$ by according to the intervention rule.

In general, for an usual body, by logic and practice, we found that $\rho_{2} \leq \rho_{3} \leq \rho_{1}$ and that $\rho_{1}$ nears to $(\sqrt{5}-1) / 2$ $\approx 0.618$ and that $\rho_{2}$ nears to $\rho_{1}^{2} \approx 1-(\sqrt{5}-1) / 2$ $\approx 0.382$ and that $\rho_{3}$ nears to $\left(1-\rho_{1}\right) /\left(2 \rho_{2}\right) \approx 1 / 2$ $=0.5$. Interestingly, they near to the golden numbers.

Theorem 3.2. Suppose that a steady multilateral system $V$ which has energy and capability of self-protection is with both intervention reaction coefficients and selfprotection coefficient $\rho_{1}, \rho_{2}$ and $\rho_{3}$. If the capability of self-protection wants to restore both subsystems $X_{K}$ and $K_{X}$, then the following statements are true.

(1) In the case of virtual disease, the treatment method is to increase the energy. If an intervention force on the subsystem $X$ of steady multilateral system $V$ is implemented such that its energy $\varphi(X)$ has been changed by increment $\Delta \varphi(X)=\Delta>0$, then all five subsystems will be changed finally by the increments as follows:

$$
\begin{gathered}
\Delta \varphi(X)_{2}=\Delta \varphi(X)+\Delta \varphi(X)_{1}=\left(1-\rho_{2} \rho_{3}\right) \Delta>0, \\
\Delta \varphi\left(X_{S}\right)_{2}=\Delta \varphi\left(X_{S}\right)+\Delta \varphi\left(X_{S}\right)_{1}=\left(\rho_{1}+\rho_{2} \rho_{3}\right) \Delta>0, \\
\Delta \varphi\left(X_{K}\right)_{2}=\Delta \varphi\left(X_{K}\right)+\Delta \varphi\left(X_{K}\right)_{1}=\left(-\rho_{1}+\rho_{3}\right) \Delta,
\end{gathered}
$$




$$
\begin{gathered}
\Delta \varphi\left(K_{X}\right)_{2}=\Delta \varphi\left(K_{X}\right)+\Delta \varphi\left(K_{X}\right)_{1}=-\left(\rho_{2}-\rho_{1} \rho_{3}\right) \Delta, \\
\Delta \varphi\left(S_{X}\right)_{2}=\Delta \varphi\left(S_{X}\right)+\Delta \varphi\left(S_{X}\right)_{1}=\left(\rho_{2}-\rho_{1} \rho_{3}\right) \Delta, \\
\forall \Delta \varphi(X)=\Delta>0
\end{gathered}
$$

(2) In the case of real disease, the treatment method is to decrease the energy. If an intervention force on the subsystem $X$ of steady multilateral system $V$ is implemented such that its energy $\varphi(X)^{\prime}$ has been changed by increment $\Delta \varphi(X)^{\prime}=-\Delta<0$, then all five subsystems will be changed finally by the increments as follows:

$$
\begin{gathered}
\Delta \varphi(X)_{2}^{\prime}=\Delta \varphi(X)^{\prime}+\Delta \varphi(X)_{1}^{\prime}=-\left(1-\rho_{2} \rho_{3}\right) \Delta<0, \\
\Delta \varphi\left(X_{S}\right)_{2}^{\prime}=\Delta \varphi\left(X_{s}\right)^{\prime}+\Delta \varphi\left(X_{S}\right)_{1}^{\prime}=-\left(\rho_{2}-\rho_{1} \rho_{3}\right) \Delta, \\
\Delta \varphi\left(X_{k}\right)_{2}^{\prime}=\Delta \varphi\left(X_{k}\right)^{\prime}+\Delta \varphi\left(X_{k}\right)_{1}^{\prime}=\left(\rho_{2}-\rho_{1} \rho_{3}\right) \Delta, \\
\Delta \varphi\left(K_{X}\right)_{2}^{\prime}=\Delta \varphi\left(K_{X}\right)^{\prime}+\Delta \varphi\left(K_{X}\right)_{1}^{\prime}=\left(\rho_{1}-\rho_{3}\right) \Delta, \\
\Delta \varphi\left(S_{X}\right)_{2}^{\prime}=\Delta \varphi\left(S_{X}\right)^{\prime}+\Delta \varphi\left(S_{X}\right)_{1}^{\prime}=-\left(\rho_{1}+\rho_{2} \rho_{3}\right) \Delta<0, \\
\forall \Delta \varphi(X)^{\prime}=-\Delta<0
\end{gathered}
$$

Corollary 3.1. Suppose that a steady multilateral system $V$ which has energy and capability of self-protection is with both intervention reaction coefficients and selfprotection coefficient $\rho_{1}, \rho_{2}$ and $\rho_{3}$. Then the capability of self-protection can make both subsystems $X_{K}$ and $K_{X}$ to be restored at the same time, i.e., the capability of self-protection is better, if and only if $\rho_{2}=\rho_{1}^{2}$ and $\rho_{3}=\rho_{1}$.

Side effects of medical problem were the question: in the medical process, destroyed the normal balance of non-fall ill system or non-intervention system. By Theorem 3.2 and Corollary 3.1, it can be seen that if the capability of self-protection of the steady multilateral system is better, i.e., the multilateral system has capability to protect all the victims to restore, then a necessary and sufficient condition is $\rho_{2}=\rho_{1}^{2}$ and $\rho_{3}=\rho_{1}$. At this point, the paper advocates the principle to avoid any side-effects of treatment.

\subsection{Mathematical Reasoning of Treatment Principle by the Neighboring Relations of Steady Multilateral Systems}

In order to show the rationality of the treatment principle above, it is needed to prove the following theorems.

Theorem 3.3. Suppose that a steady multilateral system $V$ which has energy and capability of self-protecttion is with both intervention reaction coefficients and self-protection coefficient $\rho_{1}, \rho_{2}$ and $\rho_{3}$ satisfying $\rho_{2}=\rho_{1} \rho_{3}$ and $\rho_{3}=\rho_{1}$. Then the following statements are true.
In the case of virtual disease, if an intervention force on the subsystem $X$ of steady multilateral system $V$ is implemented such that its energy $\varphi(X)$ increases the increment $\Delta \varphi(X)=\Delta>0$, then the subsystems $S_{X}$, $X_{K}$ and $K_{X}$ can be restored at the same time, but the subsystems $X$ and $X_{S}$ will increase their energies by the finally increments $\Delta \varphi(X)_{2}=\left(1-\rho_{2} \rho_{3}\right) \Delta \varphi(X)$ $=\left(1-\rho_{1}^{3}\right) \Delta$, and $\Delta \varphi\left(X_{S}\right)_{2}=\left(\rho_{1}+\rho_{2} \rho_{3}\right) \Delta \varphi(X)=$ $\left(\rho_{1}+\rho_{1}^{3}\right) \Delta$, respectively.

On the other hand, in the case of real disease, if an intervention force on the subsystem $X$ of steady multilateral system $V$ is implemented such that its energy $\varphi(X)^{\prime}$ decreases, i.e., by the increment $\Delta \varphi(X)^{\prime}=$ $-\Delta<0$, the subsystems $X_{S}, X_{K}$ and $K_{X}$ can also be restored at the same time, and the subsystems $X$ and $S_{X}$ will decrease their energies, i.e., by the finally increments $\Delta \varphi(X)_{2}=\left(1-\rho_{2} \rho_{3}\right) \Delta \varphi(X)^{\prime}=-\left(1-\rho_{1}^{3}\right) \Delta$, and $\Delta \varphi\left(S_{X}\right)_{2}=\left(\rho_{1}+\rho_{2} \rho_{3}\right) \Delta \varphi(X)^{\prime}=-\left(\rho_{1}+\rho_{1}^{3}\right) \Delta$, respectively.

Theorem 3.4. For a steady multilateral system $V$ which has energy and capability of self-protection, assume both intervention reaction coefficients and selfprotection coefficient is are $\rho_{1}, \rho_{2}$ and $\rho_{3}$ which satisfy $\rho_{2}=\rho_{1}^{2}, \quad \rho_{3}=\rho_{1}$ and $\rho_{1} \geq \rho_{0}$ where $\rho_{0}=$ 0.5897545123 (the following the same) is the solution of $2 \rho_{1}^{3}+\rho_{1}=1$. Then the following statements are true.

(1) If an intervention force on the subsystem $X$ of steady multilateral system $V$ is implemented such that its energy $\varphi(X)$ has been changed by increment $\Delta \varphi(X)=\Delta>0$, then the final increment $\left(\rho_{1}+\rho_{1}^{3}\right) \Delta$ of the energy $\varphi\left(X_{S}\right)$ of the subsystem $X_{S}$ changed is greater than or equal to the final increment $\left(1-\rho_{1}^{3}\right) \Delta$ of the energy $\varphi(X)$ of the subsystem $X$ changed based on the capability of self-protection.

(2) If an intervention force on the subsystem $X$ of steady multilateral system $V$ is implemented such that its energy $\varphi(X)$ has been changed by increment $\Delta \varphi(X)=-\Delta<0$, then the final increment $-\left(\rho_{1}+\rho_{1}^{3}\right)$ $\Delta$ of the energy $\varphi\left(S_{X}\right)$ of the subsystem $S_{X}$ changed is less than or equal to the final increment $-\left(1-\rho_{1}^{3}\right) \Delta$ of the energy $\varphi(X)$ of the subsystem $X$ changed based on the capability of self-protection.

Corollary 3.2. For a steady multilateral system $V$ which has energy and capability of self-protection, both intervention reaction coefficients and self-protection coefficient are $\rho_{1}, \rho_{2}$ and $\rho_{3}$ which satisfy $\rho_{2}=\rho_{1}^{2}, \rho_{3}=\rho_{1}$ and $\rho_{1}<\rho_{0}$. Then the following statements are true.

(1) If an intervention force on the subsystem $X$ of steady multilateral system $V$ is implemented such that its energy $\varphi(X)$ has been changed by increment $\Delta \varphi(X)=\Delta>0$, then the final increment $\left(\rho_{1}+\rho_{1}^{3}\right) \Delta$ of the energy $\varphi\left(X_{S}\right)$ of the subsystem $X_{S}$ changed is 
less than the final increment $\left(1-\rho_{1}^{3}\right) \Delta$ of the energy $\varphi(X)$ of the subsystem $X$ changed based on the capability of self-protection.

(2) If an intervention force on the subsystem $X$ of steady multilateral system $V$ is implemented such that its energy $\varphi(X)$ has been changed by increment $\Delta \varphi(X)=-\Delta<0$, then the final increment $-\left(\rho_{1}+\rho_{1}^{3}\right) \Delta$ of the energy $\varphi\left(S_{X}\right)$ of the subsystem $S_{X}$ changed is greater than the final increment $-\left(1-\rho_{1}^{3}\right) \Delta$ of the energy $\varphi(X)$ of the subsystem $X$ changed based on the capability of self-protection.

\subsection{Mathematical Reasoning of Treatment Principle by the Alternate Relations of Steady Multilateral Systems}

In order to show the rationality of the treatment principle above, it is needed to prove the following theorem.

Theorem 3.5. Suppose that a steady multilateral system $V$ which has energy and capability of self-protection is with both intervention reaction coefficients and self-protection coefficient $\rho_{1}, \rho_{2}$ and $\rho_{3}$ satisfying $\rho_{2}=\rho_{1}^{2}$ and $\rho_{3}=\rho_{1}$. Then the following statements are true.

Assume there are two subsystems $X$ and $X_{K}$ of $V$ with an alternate relation such that $X$ encounters virtual disease, and at the same time, $X_{K}$ befalls real disease. If an intervention force on the subsystem $X$ of steady multilateral system $V$ is implemented such that its energy $\varphi(X)$ has been changed by increment $\Delta \varphi(X)=\Delta>0$ and at the same time, another intervention force on the subsystem $X_{K}$ of steady multilateral system $V$ is also implemented such that its energy $\varphi\left(X_{K}\right)$ has been changed by increment $\Delta \varphi\left(X_{K}\right)=-\Delta<0$, then all other subsystems: $S_{X}, K_{X}$ and $X_{S}$ can be restored at the same time, and the subsystems $X$ and $X_{K}$ will increase and decrease their energies by the same size but the direction opposite, i.e., by the finally increments $\Delta \varphi(X)_{3}=\left(1-\rho_{2} \rho_{3}\right) \Delta=\left(1-\rho_{1}^{3}\right) \Delta>0$, and $\Delta \varphi\left(X_{K}\right)_{3}$ $=-\left(1-\rho_{2} \rho_{3}\right) \Delta=-\left(1-\rho_{1}^{3}\right) \Delta<0$, respectively.

Assume there are two subsystems $X$ and $K_{X}$ of $V$ with an alternate relation such that $X$ encounters real disease, and at the same time, $K_{X}$ befalls virtual disease. If an intervention force on the subsystem $X$ of steady multilateral system $V$ is implemented such that its energy $\varphi(X)$ has been changed by increment $\Delta \varphi(X)=-\Delta<0$, and at the same time, another intervention force on the subsystem $K_{X}$ of steady multilateral system $V$ is also implemented such that its energy $\varphi\left(K_{X}\right)$ has been changed by increment $\Delta \varphi\left(K_{X}\right)=\Delta>0$, then all other subsystems: $S_{X}, X_{k}$ and $X_{S}$ can be restored at the same time, and the subsystems $X$ and $K_{X}$ will decrease and increase their energies by the same size but the direction opposite, i.e., by the finally incre- ments $\Delta \varphi(X)_{3}=-\left(1-\rho_{2} \rho_{3}\right) \Delta=-\left(1-\rho_{1}^{3}\right) \Delta<0$, and $\Delta \varphi\left(K_{X}\right)_{3}=\left(1-\rho_{2} \rho_{3}\right) \Delta=\left(1-\rho_{1}^{3}\right) \Delta>0$, respectively.

\section{Rationality of Treatment Principle of TCM}

\subsection{Treatment Principle if Only One Organ of the Human Body System Falls Ill}

In the paper [4], it is proved that the following statements are true.

(1) If only one subsystem falls ill, mainly the treatment method should be to intervene it indirectly for case: the capability coefficient $\rho_{1} \geq \rho_{0}$ of intervention reaction, according to the treatment principle of "Real disease is to rush down his son but virtual disease is to fill his mother".

(2) The intervention method directly can be used in case $\rho_{1}<\rho_{0}$ but should be used as little as possible.

We see: The (1) is such an intervention when the intervention system was non-disease, but the incidence of law by this system will be to disease if the incidence of the disease continues to develop. The method means the idea of "Do not treat the disease after it has occurred. But treat the disease before it will occur".

Above are the main treatments. But also need to add adjuvant therapies because it is difficult to judge $\rho_{1} \geq \rho_{0}$.

In the case of virtual disease of $X$, by Theorem 3.1, the virtual disease of the loving subsystem $S_{X}$ will first occur. Therefore, the primary treatment is to increase the energy of $S_{X}$ before the virtual disease of $S_{X}$ will occur. The next more serious illness which will occur is real disease of $X_{K}$ because $X_{K}$ will insult $X$ if the disease of $X$ continues to develop. By Theorem 3.5, the second treatment method of "Strong inhibition of the same time, support the weak" should be done for $X$ and $X_{K}$ as follows:

(3) Increase the energy of $X$, and at the same time, decrease the energy of $X_{K}$ as the adjuvant therapy of increasing the energy of $S_{X}$.

The method of the adjuvant therapy also means the idea of "Do not treat the disease after it has occurred. But treat the disease before it will occur " since now the $X_{K}$ doesn't fall ill yet but it will be incidence.

In the case of real disease of $X$, by Theorem 3.1, the virtual disease of the loving subsystem $X_{S}$ will first occur. Therefore, the primary treatment is to decrease the energy of $X_{S}$ before the real disease of $X_{S}$ will occur. The next more serious illness which will occur is virtual disease of $K_{X}$ because $X$ will insult $K_{X}$ if the disease of $X$ continues to develop. By Theorem 3.5, the treatment method of "Strong inhibition of the same time, support the weak" should be done for $X$ and $K_{X}$ as follows: 
(4) Decreases the energy of $X$, and at same time, increases the energy of $K_{X}$ as the adjuvant therapy of decreasing the energy of $X_{S}$.

The method of the adjuvant therapy also means the idea of "Do not treat the disease after it has occurred. But treat the disease before it will occur" since now the $K_{X}$ doesn't fall ill yet but it will be incidence.

Above methods of the adjuvant therapy always can be done for any intervention reaction coefficient $\rho_{1}$. By Theorems 3.2 and 3.5, this methods of the adjuvant therapy will be better if intervention reaction coefficient $\rho_{2} \leq \rho_{3} \leq \rho_{1}<\rho_{0}$ because the increment $\left(1-\rho_{2} \rho_{3}\right) \Delta$ will be larger.

The intervention method can be to maintain the balance of human body system because only both disease organs and intervention organs are treated according to the normal $W u$-Xing order of human body, by Theorems 3.3 and 3.5, such that there is not any side effect for all other organs.

The treatment method also increases the capabilities of intervention reaction and self-protection because the method has used them to treat disease. The function of human tissue, the stronger the more you use. Therefore the method will maintain the balance of the five subsystems of human tissue and makes the $\rho_{1}$ and $\rho_{3}$ near to 1 , at the same time, the $\rho_{2}$ near to $\rho_{1}^{2}$. The state $\rho_{1}=\rho_{3}=1$ is the best state of the human body system. On the way, it almost has none medical and drug resistance problem since any medicine is possible good for some large $\rho_{1}, \rho_{2}$ and $\rho_{3}$.

\subsection{Treatment Principle if Only Two Organs with the Loving Relation of the Human Body System Encounter Sick}

1) Suppose that the two organs $X$ and $X_{S}$ of the human body system are abnormal (or disease). In the human body of relations between two non-compatible relations with the constraints, only two normal situations may occur:

(1) X encounters virtual disease, and at the same time, $X_{S}$ befalls virtual disease, i.e., the energy of $X$ is too low and so is the energy of $X_{S}$.

(2) $X$ encounters real disease, and at the same time, $X_{S}$ befalls real disease, i.e., the energy of $X$ is too high and so is the energy of $X_{S}$.

In paper [4], it has be shown that if intervention reaction coefficients satisfy $\rho_{3}=\rho_{1}, \rho_{2}=\rho_{1}^{2}$ and $\rho_{1} \geq \rho_{0}$, then the following statements are true.

(1) If one wants to treat the abnormal organs $X$ and $X_{S}$ for virtual disease, then the one should intervene the organ $X$ directly by increasing its energy. It means that "Virtual disease is to fill his mother".
(2) If one wants to treat the abnormal organs $X$ and $X_{S}$ for real disease, then the one should intervene organ $X_{S}$ directly by decreasing its energy. It means that "Real disease is to rush down his son".

Above are the main treatments. But also need to add adjuvant therapies because it is difficult to judge that $\rho_{3}=\rho_{1}, \rho_{2}=\rho_{1}^{2}$ and $\rho_{1} \geq \rho_{0}$.

In the case of virtual disease, since the diseases of the loving subsystems $X_{S}$ and $X$ have occurred, by Theorem 3.1, the next more serious illness which will occur is real disease of $K_{X}$ because $K_{X}$ will insult $X_{S}$ if the disease continues to develop. By Theorem 3.5, since the method of increasing the energy of $X$ has been done and a hope is that this method also can increase the energy of $X_{S}$, the treatment method of "Strong inhibition of the same time, support the weak" should be done for $X_{S}$ and $K_{X}$ as follows:

(3) Increase the energy of $X_{S}$, and at the same time, decrease the energy of $K_{X}$ as the adjuvant therapy of increasing the energy of $X$.

The method of the adjuvant therapy also means the idea of "Do not treat the disease after it has occurred. But treat the disease before it will occur" since now the $K_{X}$ doesn't fall ill yet but it will be incidence.

In the case of real disease, since the disease of the loving subsystems $X$ and $X_{S}$ have occurred, by Theorem 3.1 , the next more serious illness which will occur is virtual disease of $K_{X}$ because $X$ will insult $K_{X}$ if the disease continues to develop. By Theorem 3.5, since decrease the energy of $X_{S}$ has been done and a hope is that this method can decrease the energy of $X$, the treatment method of "Strong inhibition of the same time, support the weak" should be done for $X$ and $K_{X}$ as follows:

(4) Decrease the energy of $X$, and at the same time, increase the energy of $K_{X}$ as the adjuvant therapy of decreasing the energy of $X_{S}$.

The method of the adjuvant therapy also means the idea of "Do not treat a disease after it has occurred. But treat the disease before it will occur" since now the $K_{X}$ doesn't fall ill yet but it will be incidence.

Above methods of the adjuvant therapy always can be done for any the capability of intervention reaction coefficient $\rho_{1}$. By Theorems 3.2 - 3.5 and Corollaries 3.1 3.2 , this adjuvant therapy will be better if the intervention reaction coefficients satisfies $\rho_{2} \leq \rho_{3} \leq \rho_{1}<\rho_{0}$ because the increment $\left(1-\rho_{2} \rho_{3}\right) \Delta$ will be larger.

The intervention method also can be to maintain the balance of human body system because only both disease organs and intervention organs are treated according to the normal $W u$-Xing order of human body, by Theorems 3.2 - 3.5, such that there is not any side-effects for all other organs. 
The treatment method also increase the capability of intervention reaction because the method of using the capability of intervention reaction and capability of self-protection will maintain the balance between the five subsystems of human tissue and makes the $\rho_{1}$ and $\rho_{3}$ near to 1 , at the same time, the $\rho_{2}$ near to $\rho_{1}^{2}$. The state $\rho_{1}=\rho_{3}=1$ is the best state of the human body system. On the way, it is almost none medical and drug resistance problem since any medicine is possible good for some large $\rho_{1}$.

2) Suppose that the disease is rare disease, i.e., $X$ is real disease but $X_{S}$ is virtual disease at the same time, or $X$ is virtual disease and $X_{S}$ is real disease at the same time. In this case, the $\rho_{2} \leq \rho_{3} \leq \rho_{1}$ should be small, such as $\rho_{1}<\rho_{0}$, because the order of both loving relation and killing relation of the system has been destroyed to some extent. The treatment method of "Real disease is to rush down itself" (实则泻之) or “virtual disease is to fill itself" (虚则补之) should be directly done for both $X$ and $X_{S}$.

For the first case, if the methods of both decreasing the the energy of $X$ and increasing the energy of $X_{S}$ have been done when $\rho_{1}<\rho_{0}$, they will be to lead that $X_{K}$ kills $S_{X}$ and promotes the order of the killing relation of the multilateral system although the methods will produce a certain amount of side-effects: $\Delta \varphi\left(S_{X}\right)=$ $-\left(\rho_{1}+\rho_{2} \rho_{3}\right) \Delta<0$ and $\Delta \varphi\left(X_{K}\right)=\left(\rho_{1}+\rho_{2} \rho_{3}\right) \Delta>0$. If the above method uses the future, making the physical side-effects occur really, then it means that the ability of the intervention and self-protection capability has been restored, i.e., the $\rho_{2} \leq \rho_{3} \leq \rho_{1}$ come to larger. When $1>\rho_{1} \geq \rho_{0}$, both the main treatment and the adjuvant therapy can be used by using the following methods:

(5) Firstly decrease the energy of $K_{X}$ and increase the energy of $X_{S}$ at the same time.

(6) Secondly increase the energy of $K_{X}$ and decrease the energy of $X$ at the same time.

The method can continue to promote the $\rho_{1}$ because the capabilities of both intervention reaction and selfprotection have been used.

For the second case, if the methods of both increasing the energy of $X$ and decreasing the energy of $X_{S}$ have been done when $\rho_{1}<\rho_{0}$, there doesn't exist any sideeffects although there are only the small increments

$\Delta \varphi(X)=\left(1-2 \rho_{2} \rho_{3}-\rho_{1}\right) \Delta>0$, and $\Delta \varphi\left(X_{S}\right)=-\left(1-2 \rho_{2} \rho_{3}-\rho_{1}\right) \Delta<0$.

If the above method uses the future, making the more serious disease occur really, then it means that the capabilities of both the intervention reaction and self-protecttion have been restored, i.e., $\left(1-2 \rho_{2} \rho_{3}-\rho_{1}\right)<0$, or the case: $\rho_{2}=\rho_{1}^{2}, \rho_{3}=\rho_{1}$ and $\rho_{1} \geq \rho_{0}$. In this time, the adjuvant therapy can be used by using the following methods:
(7) Increase the energy of $S_{X}$ and decrease the energy of $X_{K}$ at the same time, as the adjuvant therapy of increasing the energy of $X$ and decreasing the energy of $X_{S}$.

The method can continue to promote the $\rho_{1}$ because the capabilities of both intervention reaction and self-protection have been used. But the method should be used as little as possible because it may be to lead that $S_{X}$ will insult $X_{K}$ and destroys the killing order of the human body system if it always keen to be used.

\subsection{Treatment Principle if Only Two Organs with the Killing Relation of the Human Body System Encounter Sick}

1) Suppose that the organs $X$ and $X_{K}$ of a human body system are abnormal (or disease). In the human body system of relations between two non-compatible relations with the constraints, only a serious disease may occur: $X$ encounters virtual disease, and at the same time, $X_{K}$ befalls real disease, i.e., the energy of $X$ is too low and the energy of $X_{K}$ is too high. Such a disease has a serious problem because $X_{K}$ insults $X$. By Theorem 3.1, the disease most likely to lead to more serious illnesses: the virtual disease of $S_{X}$ and the real disease of $K_{X}$.

In paper [4], it has been shown that if intervention reaction coefficients satisfy $\rho_{2}=\rho_{1}^{2}$, then one should intervene organ $X$ directly by increasing its energy, and at the same time, intervene organ $X_{K}$ directly by decreasing its energy if the one wants to treat the abnormal organs $X$ and $X_{K}$. It means that"Strong inhibition of the same time, support the weak".

Of course, a method of the adjuvant therapy always can be done as follows:

(1) Increase the energy of $S_{X}$ and decrease the energy of $K_{X}$ at the same time. It means that "Virtual disease is to fill his mother but real disease is to rush down his son".

The method can continue to promote the $\rho_{1}$ because the capabilities of both intervention reaction and selfprotection have been used.

2) If a less serious disease has occurred: $X$ encounters real disease, and at the same time, $X_{K}$ befalls virtual disease, i.e., the energy of $X$ is too high and the energy of $X_{K}$ is too low. Such a disease has not a serious problem in this time yet, but by Theorem 3.1 the disease most likely to lead to more serious illnesses: the virtual disease of $K_{X}$ and the real disease of $X_{S}$. Therefore, this disease should be done to treat as more serious diseases.

The primary treatment method of "Strong inhibition of the same time, support the weak" should be done for $X$, $X_{K}$ and $K_{X}$, i.e., to decrease the energy of $X$, and at the same time, to increase the energies of $X_{K}$ and $K_{X}$. 
The method also means the idea of "Do not treat a disease after it has occurred. But treat the disease before it will occur". It is because the $K_{X}$ is not sick at this time, but it will be incidence. And secondary treatment is as follows:

(2) Decrease the energy of the subsystem $X_{S}$. It means that "Real disease is to rush down his son".

Above methods of the adjuvant therapy always can be done for any intervention reaction coefficient $\rho_{1}$. By Theorems 3.2 - 3.5 and Corollaries 3.1 - 3.2, the methods of the adjuvant therapy will be better if the intervention reaction coefficients satisfies $\rho_{2} \leq \rho_{3} \leq \rho_{1}<\rho_{0}$ because at this time the increment $\left(1-\rho_{2} \rho_{3}\right) \Delta$ was larger.

The intervention method can be to maintain the balance of human body system because only both disease organs and intervention organs are treated according to the normal Wu-Xing order of human body, by using Theorems $3.2-3.5$, such that there is not any side-effects for all other organs.

The treatment method also increase the capability of intervention reaction because the method of using both the capability of intervention reaction and capability of self-protection will maintain the balance between the five subsystems of human tissue and makes the $\rho_{1}$ and $\rho_{3}$ near to 1 , at the same time, the $\rho_{2}$ near to $\rho_{1}^{2}$. The state $\rho_{1}=\rho_{3}=1$ is the best state of the human body system. On the way, it is almost none medical and drug resistance problem since any medicine is possible good for some large $\rho_{1}$.

3) Suppose that the disease is rare disease, i.e., $X$ is real disease and so is $X_{K}$ at the same time, or if $X$ is virtual disease and so is $X_{K}$ at the same time. In this case, the $\rho_{2} \leq \rho_{3} \leq \rho_{1}$ should be small, such as $\rho_{1}<\rho_{0}$, because the order of both the loving relation and the killing relation of the system has been destroyed to some extent. The directly treating method of "Real disease is to rush down itself” (实则泻之) or “virtual disease is to fill itself" (虚则补之) should be directly done for $X$ and $X_{K}$.

For the first case, if the methods of decreasing the energies of both $X$ and $X_{K}$ have been done when $\rho_{1}<\rho_{0}$, the methods will produce a certain amount of sideeffects: $\Delta \varphi\left(X_{S}\right)=-\left(\rho_{1}+\rho_{2} \rho_{3}\right) \Delta<0$ and $\Delta \varphi\left(S_{X}\right)=$ $-\left(\rho_{1}+\rho_{2} \rho_{3}\right) \Delta<0$. If the above method uses the future, making the physical side-effects occur, then it means that the ability of the intervention and self-protection capability has been restored, i.e., the $\rho_{2} \leq \rho_{3} \leq \rho_{1}$ comes to larger. When $\rho_{1} \geq \rho_{0}$, the adjuvant therapy can be used by using the following methods:

(3) Decrease the energies of $X_{S}$ and $K_{X}$ at the same time. It means that "Virtual disease is to fill his mother but real disease is to rush down his son".
The method can continue to promote the $\rho_{1}$ because the capabilities of both intervention reaction and selfprotection have been used.

For the second case, if the methods of increasing the energies of both $X$ and $X_{K}$ have been done when $\rho_{1}<\rho_{0}$, the methods will produce a certain amount of sideeffects: $\Delta \varphi\left(X_{S}\right)=\left(\rho_{1}+\rho_{2} \rho_{3}\right) \Delta>0$ and $\Delta \varphi\left(K_{X}\right)=$ $\left(\rho_{1}+\rho_{2} \rho_{3}\right) \Delta>0$. If the above method uses the future, making the physical side-effects occur, then it means that the ability of the intervention and self-protection capability has been restored, i.e., the $\rho_{2} \leq \rho_{3} \leq \rho_{1}$ come to larger. When $\rho_{1} \geq \rho_{0}$, the adjuvant therapy can be used by using the following methods:

(4) Increase the energies of $S_{X}$ and $X_{S}$ at the same time. It means that "Virtual disease is to fill his mother but real disease is to rush down his son".

The method can continue to promote the $\rho_{1}$ because the capabilities of both intervention reaction and selfprotection have been used.

\section{Conclusions}

This work shows how to treat the diseases of a human body system and a lot of methods are presented.

If only one organ falls ill, the treatment method should be to intervene it indirectly by using primary treatment and secondary treatment.

(1) In the case of virtual disease of $X$, the primary treatment is to increase the energy of the loved subsystem $S_{X}$. And the secondary treatment is to increase the energy of $X$, and at the same time, to decrease the energy of $X_{K}$ as the adjuvant therapy of increasing the energy of $S_{X}$.

(2) In the case of real disease of $X$, the primary treatment is to decrease the energy of the loving subsystem $X_{S}$. And the secondary treatment is to decrease the energy of $X$, and at the same time, to increase the energy of $K_{X}$ as the adjuvant therapy of decreasing the energy of $X_{S}$.

If only two organs $X$ and $X_{S}$ of the human body system fall ill, the treatment method should be to intervene it indirectly by using primary treatment and secondary treatment.

(1) Suppose that $X$ encounters virtual disease, and at the same time, $X_{S}$ befalls virtual disease. The primary treatment is to increase the energy of the subsystem $X$. And secondary treatment is to increase the energy of $X_{S}$, and at the same time, to decrease the energy of $K_{X}$ as the adjuvant therapy of increasing the energy of $X$.

(2) Suppose that $X$ encounters real disease, and at the same time, $X_{S}$ befalls real disease. The primary treatment is to decrease the energy of the subsystem $X_{S}$. 
And secondary treatment is to decrease the energy of $X$, and at the same time, to increase the energy of $K_{X}$ as the adjuvant therapy of increasing the energy of $X$.

(3) Suppose that $X$ encounters real disease, and at the same time, $X_{S}$ befalls virtual disease (rare disease). The mainly treatment is to decrease the energy of the subsystem $X$, and at the same time, to increase the energy of the subsystem $X_{S}$ if $\rho_{1}<\rho_{0}$. When $1>\rho_{1} \geq \rho_{0}$, the adjuvant therapy is firstly to decrease the energy of $K_{X}$ and to increase the energy of $X_{S}$ at the same time; and secondly to increase the energy of $K_{X}$ and to decrease the energy of $X$ at the same time.

(4) Suppose that $X$ encounters virtual disease, and at the same time, $X_{S}$ befalls real disease (rare disease). The primary treatment is to increase the energy of the subsystem $X$, and at the same time, to decrease the energy of the subsystem $X_{S}$ if $\rho_{1}<\rho_{0}$. When $\rho_{1} \geq \rho_{0}$, the secondary treatment is to increase the energy of $S_{X}$ and to decrease the energy of $X_{K}$ at the same time, as the adjuvant therapy of increasing the energy of $X$ and decreasing the energy of $X_{S}$.

If only two organs $X$ and $X_{K}$ of the human body system fall ill, the treatment method should be to intervene it indirectly by using primary treatment and secondary treatment.

(1) Suppose that $X$ encounters virtual disease, and at the same time, $X_{K}$ befalls real disease. The primary treatment is to increase the energy of the subsystem $X$, and at the same time, to decrease the energy of the subsystem $X_{K}$. And secondary treatment is to increase the energy of the subsystem $S_{X}$ and to decrease the subsystem $K_{X}$ at the same time.

(2) Suppose that $X$ encounters real disease, and at the same time, $X_{K}$ befalls virtual disease (may be to occur more serious disease). The primary treatment is to decrease the energy of the subsystem $X$, and at the same time, to increase the energies of both $X_{K}$ and $K_{X}$. And secondary treatment is to decrease the energy of the subsystem $X_{S}$.

(3) Suppose that $X$ encounters real disease, and at the same time, $X_{K}$ be falls real disease (rare disease). The mainly treatment is to decrease the energies of the subsystems $X$ and $X_{K}$ if $\rho_{1}<\rho_{0}$. When $\rho_{1} \geq \rho_{0}$, the adjuvant therapy is to decrease the energies of $X_{S}$ and $K_{X}$ at the same time.

(4) Suppose that $X$ encounters virtual disease, and at the same time, $X_{K}$ befalls virtual disease (rare disease). The mainly treatment is to increase the energies of the subsystems $X$ and $X_{K}$ if $\rho_{1}<\rho_{0}$. When $\rho_{1} \geq \rho_{0}$, the adjuvant therapy is to increase the energies of $S_{X}$ and $X_{S}$ at the same time.

Other properties, such as the order of “Wu Xing" (五 行), “Wu Yun Liu Qi” (五运六气), “Zang Xiang” (藏象),
"Jing Luo" (经络), and so on, will be discussed in the next articles.

\section{Acknowledgements}

This article has been repeatedly invited as reports, such as people's University of China in medical meetings, Shanxi University, Xuchang College, and so on. The work was supported by Specialized Research Fund for the Doctoral Program of Higher Education of Ministry of Education of China (Grant No. 44k55050).

\section{References}

[1] Y. S. Zhang, "Multilateral Matrix Theory," 1993. http://www.mlmatrix.com

[2] Y. S. Zhang, "Theory of Multilateral Systems," 2007. http://w ww.mlmatrix.com

[3] X. P. Chen, W. J. Zhu, C. Y. Pan and Y. S. Zhang, "Multilateral System," Journal of Systems Science, Vol. 17, No. 1, 2009, pp. 55-57.

[4] Y. S. Zhang, "Mathematical Reasoning of Treatment Principle Based on 'Yin Yang Wu Xing' Theory in Traditional Chinese Medicine," Chinese Medicine, Vol. 2, No. 1, 2011, pp. 6-15. doi:10.4236/cm.2011.21002

[5] Y. S. Zhang, "Mathematical Reasoning of Treatment Principle Based on the Stable Logic Analysis Model of Complex Systems," Intelligent Control and Automation, Vol. 2, No. 4, 2011, in press.

[6] Y. S. Zhang, S. S. Mao, C. Z. Zhan and Z. G. Zheng, "Stable Structure of the Logic Model with Two Causal Effects," Chinese Journal of Applied Probability and Statistics, Vol. 21, No. 4, 2005, pp. 366-374.

[7] N. Q. Feng, Y. H. Qiu, F. Wang, Y. S. Zhang and S. Q. Yin, "A Logic Analysis Model about Complex System's Stability: Enlightenment from Nature," Lecture Notes in Computer Science, Vol. 3644, 2005, pp. 828-838. doi:10.1007/11538059 86

[8] N. Q. Feng, Y. H. Qiu, Y. S. Zhang, F. Wang and Y. He, "A Intelligent Inference Model about Complex System's Stability: Inspiration from Nature," International Journal of Intelligent Technology, Vol. 1, 2005, pp. 1-6.

[9] N. Q. Feng, Y. H. Qiu, Y. S. Zhang, C. Z. Zhan and Z. G. Zheng, "A Logic Analysis Model of Stability of Complex System Based on Ecology," Computer Science, Vol. 33, No. 7, 2006, pp. 213-216.

[10] Y. S. Zhang and S. S. Mao, "The Origin and Development Philosophy Theory of Statistics," Statistical Research, Vol. 12, 2004, pp. 52-59.

[11] C. Y. Pan, X. P. Chen, Y. S. Zhang and S. S. Mao, "Logical Model of Five-Element Theory in Chinese Traditional Medicine," Journal of Chinese Modern Traditional Chinese Medicine, Vol. 4, No. 3, 2008, pp. 193-196.

[12] C. Luo and Y. S. Zhang, "Framework Definition and Partition Theorems Dealing with Complex Systems: One 
of the Series of New Thinking," Journal of Shanghai Instituie of Thennolegy (Natural Science), Vol. 10, No. 2, 2010, pp. 109-114.

[13] C. Luo and Y. S. Zhang, "Framework and Orthogonal Arrays: The New Thinking of Dealing with Complex Systems Series Two," Journal of Shanghai Institute of Thennolegy (Natural Science), Vol. 10, No. 3, 2010, pp. 159-163.

[14] C. Luo, X. P. Chen and Y. S. Zhang, "The Turning Point Analysis of Finance Time Series," Chinese Journal of Applied Probability and Statistics, Vol. 26, No. 4, 2010, pp. 437-442

[15] Y. S. Zhang, X. Q. Zhang and S. Y. Li, "SAS Language Guide and Application," Shanxi People's Press, Shanxi, 2011.

[16] Y. S. Zhang, S. Q. Pang, Z. M. Jiao and W. Z. Zhao, "Group Partition and Systems of Orthogonal Idempotents," Linear Algebra and Its Applications, Vol. 278, 1998, pp. 249-262.

[17] C. Y. Pan, H. N. Ma, X. P. Chen and Y. S. Zhang, "Proof Procedure of Some Theories in Statistical Analysis of Global Symmetry," Journal of East China Normal University (Natural Science), Vol. 142, No. 5, 2009, pp. $127-137$

[18] Y. S. Zhang, Y. Q. Lu and S. Q. Pang, "Orthogonal Arrays Obtained by Orthogonal Decomposition of Projection Matrices," Statistica Sinica, Vol. 9, 1999, pp. 595604.

[19] Y. S. Zhang, S. Q. Pang and Y. P. Wang, "Orthogonal Arrays Obtained by Generalized Hadamard Product," Discrete Mathematics, Vol. 238, 2001, pp. 151-170. doi:10.1016/s0012-365x(00)00421-0

[20] Y. S. Zhang, L. Duan, Y. Q. Lu and Z. G. Zheng, "Construction of Generalized Hadamard Matrices," Journal of Statistical Planning, Vol. 104, 2002, pp. 239-258. doi:101016/s0378-3758(01)00249-x

[21] Y. S. Zhang, "Orthogonal Arrays Obtained by Repeating-Column Difference Matrices," Discrete Mathematics,
Vol. 307, No. 2, 2007, pp. 246-261. doi:10.1016/j.disc.2006.06.029

[22] Y. S. Zhang, "Data Analysis and Construction of Orthogonal Arrays," East China Normal University, Shanghai, 2006.

[23] X. D. Wang, Y. C. Tang, X. P. Chen and Y. S. Zhang, "Design of Experiment in Global Sensitivity Analysis Based on ANOVA High-Dimensional Model Representation," Communications in Statistics-Simulation and Computation, Vol. 39, No. 6, 2010, pp. 1183-1195. doi:10.1080/03610918.2010.484122

[24] J. Y. Liao, J. J. Zhang, Y. S. Zhang, "Robust Parameter Design on Launching an Object to Goal," Mathematics in Practice and Theory, Vol. 40, No. 24,.2010, pp. 126-132

[25] X. Q. Zhang, Y. S. Zhang and S. S. Mao, "Statistical Analysis of 2-Level Orthogonal Satursted Designs: The Procedure of Searching Zero Effects," Journal of East China Normal University (Natural Science), Vol. 24, No. 1, 2007, pp. 51-59

[26] Y. S. Zhang, W. G. Li, S. S. Mao and Z. G. Zheng, "Orthogonal Arrays Obtained by Generalized Difference Matrices with g Levels," Science China Mathematics, Vol. 54, No. 1, 2011, pp. 133-143. doi:10.1007/s11425-010-4144-y

[27] J. T. Tian, Y. S. Zhang, Z. Q. Zhang, C. Y. Pan and Y. Y. Gan, "The Comparison and Application of Balanced Block Orthogonal Arrays and Orthogonal Arrays," Journal of Mathematics in Practice and Theory, Vol. 39, No. 22, 2009, pp. 59-67.

[28] C. Luo and C. Y. Pan, "Method of Exhaustion to Search Orthogonal Balanced Block Designs," Chinese Journal of Applied Probability and Statistics, Vol. 27, No. 1, 2011, pp. 1-13.

[29] Research Center for Chinese and Foreign Celebrities and Developing Center of Chinese Culture Resources, "Chinese Philosophy Encyclopedia," Shanghai People Press, Shanghai, 1994. 